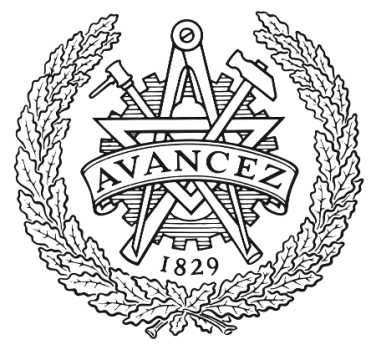

CHALMERS

UNIVERSITY OF TECHNOLOGY

\title{
LC-MS/MS Quantification Reveals Ample Gut Uptake and Metabolization of Dietary Phytochemicals in Honey Bees (Apis mellifera)
}

Downloaded from: https://research.chalmers.se, 2023-04-26 14:55 UTC

Citation for the original published paper (version of record):

Hjort Vidkjaer, N., Fomsgaard, I., Kryger, P. (2021). LC-MS/MS Quantification Reveals Ample Gut Uptake and Metabolization of Dietary Phytochemicals

in Honey Bees (Apis mellifera). Journal of Agricultural and Food Chemistry, 69(2): 627-637.

http://dx.doi.org/10.1021/acs.jafc.0c03584

N.B. When citing this work, cite the original published paper. 


\title{
LC-MS/MS Quantification Reveals Ample Gut Uptake and Metabolization of Dietary Phytochemicals in Honey Bees (Apis mellifera)
}

\author{
Nanna Hjort Vidkjær,* Inge S. Fomsgaard, and Per Kryger
}

Cite This: J. Agric. Food Chem. 2021, 69, 627-637

Read Online

ACCESS | L W Metrics \& More | 国 Article Recommendations | sl Supporting Information

ABSTRACT: The honey bee pollen/nectar diet is rich in bioactive phytochemicals and recent studies have demonstrated the potential of phytochemicals to influence honey bee disease resistance. To unravel the role of dietary phytochemicals in honey bee health it is essential to understand phytochemical uptake, bioavailability, and metabolism but presently limited knowledge exists. With this study we aim to build a knowledge foundation. For 5 days, we continuously fed honey bees on eight individual phytochemicals and measured the concentrations in whole and dissected bees by HPLC-MS/MS. Ample phytochemical metabolization was observed, and only $6-30 \%$ of the consumed quantities were recovered. Clear differences in metabolization rates were evident, with atropine, aucubin, and triptolide displaying significantly slower metabolism. Phytochemical gut uptake was also demonstrated, and oral bioavailability was $4-31 \%$, with the highest percentages observed for amygdalin, triptolide, and aucubin. We conclude that differences in the chemical properties and structure impact phytochemical uptake and metabolism.

KEYWORDS: honey bee, Apis mellifera, senkirkine, senecionine, gelsemine, amygdalin, atropine, methyllycaconitine, triptolide, aucubin, HPLC-MS/MS, quantification, diet, gut, uptake, metabolization, bioavailability, phytochemical

\section{INTRODUCTION}

Honey bees (Apis mellifera) are important pollinators of many food crops, and the worldwide decline in honey bees and other insect pollinators raises concern for our food production. ${ }^{1,2}$ Xenobiotics, diseases, parasites, and changing floral resources, resulting from intensified land use for farming, are proposed as the major factors responsible for the decline. ${ }^{2-6}$ These factors influence honey bee populations individually as well as in synergy. ${ }^{3-5}$ Honey bees subjected to certain xenobiotics are, for instance, more susceptible to specific diseases, and the parasitic mite, Varroa destructor, is an important factor in the transmission of viruses. $^{7-9}$ The effects of environmental changes and the resulting changes in floral resources have been less intensely studied compared to other factors. Floral resources in the landscape surrounding the hive determine the composition of the honey bees' pollen and nectar diet. Changes in floral resources, due to season, weather conditions, changes in land use, or transhumance of colonies by beekeepers, can therefore have a significant impact on the diet. It is well established that essential dietary nutrients are paramount to honey bee health..$^{10-12}$ However, pollen and nectar also contain a plethora of bioactive phytochemicals, also known as plant secondary metabolites. These compounds have been widely utilized in human medicine, but only recently have studies concerning effects of phytochemicals on honey bee health emerged. Although this is still an area of research in its infancy, these studies demonstrate the potential for phytochemicals to positively impact honey bee physiology and disease resistance, ${ }^{13-19}$ yet phytochemicals with toxic effects are also known. ${ }^{20,21}$ Collectively, these observations emphasize the need for fundamental research aiming at disentangling the role of bioactive phytochemicals in honey bee health Clarification of phytochemical uptake and metabolization processes in honey bees are essential steps toward achieving this understanding and potentially implementing healthpromoting compounds in apiculture.

The aim of this study was to establish a knowledge foundation for future detailed investigations of phytochemicals in honey bee health by obtaining new knowledge about the oral bioavailability, gut uptake, and metabolization percentages of a variety of phytochemicals naturally present in pollen and nectar. A set of compounds, whose occurrence in plants and natural concentrations in pollen and/or nectar are known, were chosen for the feeding experiment presented here: senkirkine and senecionine (pyrrolizidine alkaloids); methyllycaconitine (diterpenoid alkaloid); gelsemine (indole alkaloid); atropine (tropane alkaloid); triptolide (diterpenoid epoxide); aucubin (iridoid glycoside); and amygdalin (cyanogenic glycoside). All of these compounds have a history of occurrence in honey bee nectar and pollen, and they have all been singled out for their phytochemical properties. There are also phytochemicals with potential toxic effects included in this feeding experiment, but all compounds were fed to the honey bees in concentrations

Received: June 6, 2020

Revised: November 15, 2020

Accepted: November 30, 2020

Published: January 8, 2021 
Table 1. Phytochemicals Fed to Honey Bees and Their Natural Concentrations Reported in Pollen and Nectar ${ }^{a}$

\begin{tabular}{|c|c|c|c|c|}
\hline Phytochemical & Structure & $\begin{array}{l}\text { Pollen } \\
\text { (ppm) }\end{array}$ & $\begin{array}{c}\text { Nectar } \\
(\text { ppm) }\end{array}$ & $\begin{array}{c}\text { Feeding } \\
\text { concentration } \\
(\mathbf{u g} / \mathbf{m L}=\mathbf{p p m})\end{array}$ \\
\hline Gelsemine & & No data & $5.8-246.1^{22}$ & 126 \\
\hline Senecionine & & $1700^{68}$ & $0.004-0.007^{\mathrm{b}, 23}$ & 1.3 \\
\hline Senkirkine & & $250^{68}$ & $0.002-0.004^{b, 23}$ & 1.3 \\
\hline Atropine & & No data & $0.19-37^{24}$ & 18.6 \\
\hline Aucubin & & $100^{25}$ & $3300^{25}$ & 1600 \\
\hline Amygdalin & & $1889^{26}$ & $4.9-6.7^{26}$ & 50 \\
\hline Triptolide & & No data & $0.5-0.72^{b, 27}$ & 0.2 \\
\hline Methyllycaconitine & & No data & $0.02-0.04^{28}$ & 0.057 \\
\hline
\end{tabular}

${ }^{a}$ Botanical sources of the individual compounds are listed in Table S1. ${ }^{b}$ Concentrations measured in honey. No data available for nectar.

within the range naturally found in nectar and pollen and below toxicity thresholds.

\section{MATERIALS AND METHODS}

Chemicals. Organic solvents used for extractions were HPLC grade obtained from Rathburn (Mikrolab, Aarhus, Denmark), except for ethanol (96\%), which was obtained from Kemetyl AB (Haninge, Sweden). Acetonitrile and methanol for LC-MS analysis was LCMS grade purchased from Fisher Scientific (Roskilde, Denmark). Analytical grade formic acid and LC-MS grade ammonium formate were obtained from Sigma-Aldrich (Copenhagen, Denmark), LC-MS grade acetic acid was obtained from VWR (Søborg, Denmark), and analytical grade ammonium hydroxide was obtained from Merck (Darmstadt, Germany). All water used was Milli-Q water collected from a Dionex (Hvidovre, Denmark) Milli-Q purifier. Gelsemine, senecionine, senkirkine, atropine, aucubin, and amygdalin were obtained from Extrasynthese (Genay, France). Triptolide was purchased from BioNordika (Herlev, Denmark), whereas methyllycaconitine was supplied by Sigma-Aldrich. Sucrose (>99\%) for the feeding solutions was purchased from Sigma-Aldrich. 
Table 2. Validation of the Analytical Method and MRM Transitions (Q1/Q3) Monitored for the Eight Phytochemicals

\begin{tabular}{|c|c|c|c|c|c|c|c|c|}
\hline \multirow[b]{2}{*}{ phytochemical } & \multicolumn{2}{|c|}{ MRM transition ${ }^{a}$} & \multirow[b]{2}{*}{$\begin{array}{l}\text { retention time } \\
(\mathrm{min})\end{array}$} & \multirow[b]{2}{*}{$\begin{array}{l}\text { spike level } \\
\text { (ng/bee) }\end{array}$} & \multirow[b]{2}{*}{$\begin{array}{c}\text { recovery }^{b} \\
(\%)\end{array}$} & \multirow[b]{2}{*}{$\begin{array}{l}\text { RSD } \\
(\%)\end{array}$} & \multirow[b]{2}{*}{$\begin{array}{c}\text { LOD }^{c} \\
\text { (ng/bee) }\end{array}$} & \multirow[b]{2}{*}{$\begin{array}{c}\mathrm{LOQ}^{c} \\
(\mathrm{ng} / \mathrm{bee})\end{array}$} \\
\hline & $\begin{array}{c}\text { quantifier } \\
(\mathrm{m} / \mathrm{z})\end{array}$ & $\begin{array}{c}\text { qualifier } \\
(\mathrm{m} / \mathrm{z})\end{array}$ & & & & & & \\
\hline atropine & $290 / 124$ & $290 / 77$ & 8.9 & 240 & $108 \pm 6$ & 6 & 45 & 151 \\
\hline senecionine & $336 / 120$ & $336 / 308$ & 9.1 & 4.9 & $78 \pm 7$ & 9 & 1.1 & 3.5 \\
\hline senkirkine & $366 / 168$ & $366 / 150$ & 9.9 & 7.4 & $72 \pm 5$ & 8 & 1.2 & 4 \\
\hline gelsemine & $323 / 70$ & $323 / 236$ & 7.6 & 510 & $74 \pm 3$ & 5 & 56 & 185 \\
\hline methyllycaconitine & $683 / 216$ & $683 / 651$ & 12.8 & 0.3 & $99 \pm 12$ & 12 & 0.1 & 0.4 \\
\hline amygdalin & $456 / 323$ & $456 / 59$ & 9.0 & 240 & $67 \pm 3$ & 4 & 21 & 70.2 \\
\hline aucubin & $345 / 183$ & $345 / 165$ & 5.1 & 16,400 & $76 \pm 10$ & 13 & 4809 & 16,030 \\
\hline triptolide & $378 / 361$ & $378 / 91$ & 4.8 & 4.4 & $82 \pm 82$ & 7 & 0.8 & 2.7 \\
\hline
\end{tabular}

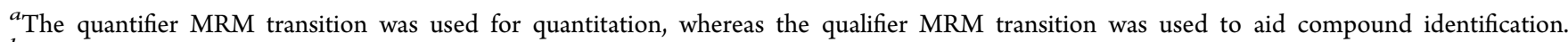

${ }^{b}$ Recovery percentages are listed as \pm the SD. ${ }^{c} \mathrm{LOD}$ and LOQ were calculated as three and ten times the SD, respectively, of the eight replicates prepared for method validation.

Feeding Experiment. Honey bees (A. mellifera L) were collected from brood frames in the apiary of Aarhus University, Flakkebjerg. The collected bees were fed on 50\% sucrose for 3 days. On day 3, the bees were divided into eight experimental groups placed in feeding cages $(N=49-73)$. The exact numbers of bees in the individual cages were counted at the end of the experiment. A portion of bees were also collected for the analysis of the presence of the compounds prior to the experiment. Thus, these bees served as a negative control group. The feeding boxes were placed in incubators in complete darkness under the following conditions: $34{ }^{\circ} \mathrm{C}$; 38-40\% relative humidity. For 5 days, the bees in the eight cages were separately fed one compound per cage at the concentrations listed in Table 1 in 50\% sucrose syrup. Structures of the tested compounds and their natural concentrations $^{22-28,68}$ are also listed in Table 1. Information about plants known to produce the phytochemicals fed to the honey bees is included in the Supporting Information (Table S1). The prepared solutions were placed in $1.5 \mathrm{~mL}$ Eppendorf tubes, and the bottom of the tubes was pierced with a sterilized needle to allow the bees to feed on the solution. The feeding solutions were replaced every $24 \mathrm{~h}$ to prevent compound degradation and measure food intake. Dead bees were counted and removed daily. On day 5 , the feeding containers were removed, and $2 \mathrm{~h}$ later, the bees were anesthetized with $\mathrm{CO}_{2}$ and killed by freezing.

Selection of Extraction Protocols and Method Validation. The extraction protocols were initially developed by spiking the individual compounds into single lyophilized and pulverized bees $(N$ $=3$ ) in an amount close to the mean daily consumption per bee of the individual compounds (Figure 2). Once methods displaying acceptable recovery percentages and repeatability were established, the bees from the experimental groups were analyzed $(N=12)$ and the mean concentrations in the bees were calculated. Then, the analytical protocols were fully validated using eight replicates (individual lyophilized and pulverized bees) by spiking an amount corresponding to the mean concentration measured in the individual bees (Table 2). Recovery percentages were evaluated according to the EURACHEM guidelines. $^{29}$ The limits of detection (LOD) and quantification (LOQ) were established by determining the standard deviation (SD) of the individual compounds in the spiked bees of the replicated recovery experiment. According to the EURACHEM guidelines, the LOD was determined as $3 \mathrm{SD}$, while the LOQ was set to $10 \mathrm{SD}$. The quantification precision was assessed as the relative SD (RSD \%) of the eight spiked replicates.

Optimized Methods for Sample Preparation and Extraction of Whole Honey Bees. Twelve individual bees from each of the experimental groups were rinsed with water and placed individually in $1.5 \mathrm{~mL}$ Eppendorf tubes. The bees were then lyophilized and transferred individually to Falcon tubes for extraction. Three metal beads were added, and the bees were pulverized by vibration for $30 \mathrm{~s}$ at $1500 \mathrm{rpm}$ using a Geno/Grinder (SPEX Sample Prep 2010, Metuchen NJ 08840). The extraction solvents were added, and the bees were extracted by shaking using an Intelli-Mixer for $1 \mathrm{~h}(60$ rpm). The extraction solvents were as follows: aucubin: $20 \mathrm{~mL}$ methanol; methyllycaconitine: $3 \mathrm{~mL} \mathrm{1:1} \mathrm{methanol/water;} \mathrm{triptolide:} 3$ $\mathrm{mL}$ methanol; senecionine: $8 \mathrm{~mL} \mathrm{7:3} \mathrm{methanol/water;} \mathrm{senkirkine:} 8$

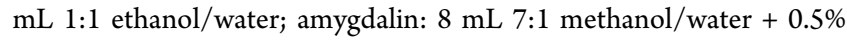
acetic acid; gelsemine: $8 \mathrm{~mL} \mathrm{1:1} \mathrm{ethanol/water,} \mathrm{and} \mathrm{atropine:} 8 \mathrm{~mL}$ $7: 3$ methanol/water $+0.5 \%$ acetic acid. After extraction, the samples were centrifuged $\left(12 \mathrm{~min}, 4{ }^{\circ} \mathrm{C}, 4500 \mathrm{rpm}\right)$. Extracts of bees fed on aucubin, methyllycaconitine, senecionine, senkirkine, amygdalin, gelsemine, and atropine were diluted to $10 \%$ organic solvent with Milli-Q water containing $0.5 \%$ acetic acid, filtered using a syringe filter (Kinesis KX PTFE syringe filter $13 \mathrm{~mm}, 0.22 \mu \mathrm{m}$, Mikrolab, Aarhus, Denmark), and analyzed by HPLC-MS/MS as described below. Extracts of bees fed on triptolide were further purified by solid phase extraction (SPE) using a protocol modified from Wang et al. ${ }^{30}$ One milliliter of the methanol extracts was diluted to $10 \mathrm{~mL}$ with Milli-Q water, $100 \mu \mathrm{L}$ of formic acid was added, and the diluted extracts were loaded onto $30 \mathrm{mg}$ Oasis HLB prime SPE (Waters, Hedehusene, Denmark) cartridges without prior column conditioning. The SPE cartridges were first washed with $1 \mathrm{~mL}$ of $2 \%$ ammonium hydroxide in 1:9 methanol/water and then with $1 \mathrm{~mL}$ of $2 \%$ acetic acid in 3:7 methanol/water, and triptolide was eluted from the cartridges with 1 $\mathrm{mL}$ of 4:1 methanol/water. Prior to HPLC-MS/MS analyses, $225 \mu \mathrm{L}$ of the SPE eluates was diluted with $275 \mu \mathrm{L}$ of $5 \mathrm{mM}$ ammonium formate and filtered using a syringe filter (Kinesis KX PTFE syringe filter $13 \mathrm{~mm}, 0.22 \mu \mathrm{m}$, Mikrolab, Aarhus, Denmark).

Sample Preparation and Extraction of Dissected Honey Bees. Six bees from each of the experimental groups were defrosted and rinsed with water, and their heads were removed with a scalpel to cut the esophagus. The complete alimentary canal (hereafter "gut") was removed by grabbing the stinger with tweezers and gently pulling until the alimentary canal was released. ${ }^{31}$ The two samples consisting of the gut and the rest of the bee without the gut (head, thorax, and abdomen; hereafter, "bee without gut") were lyophilized separately in $1.5 \mathrm{~mL}$ Eppendorf tubes. Upon drying, the samples comprising the bees without guts were transferred to the extraction Falcon tubes, pulverized, and extracted as described above for the whole bees. Samples comprising the guts were instead pulverized directly in the $1.5 \mathrm{~mL}$ Eppendorf tubes by adding two metal beads and placing these tubes in the Geno/Grinder using a modified rack. Because of the small sample size, the pulverized guts were then gradually transferred to the extraction Falcon tubes using the extraction solvents to flush the material from the Eppendorf tubes. The remaining parts of the extraction followed the protocols described above for whole bees.

HPLC-MS/MS Quantification. The sample extracts were quantified using an HPLC (1260 Infinity, Agilent Technologies, Glostrup, Denmark) coupled to a mass spectrometer (4500 QTRAP, Sciex, Copenhagen, Denmark) with electrospray ionization operated in multiple reaction monitoring mode (MRM) using nitrogen as the source and collision gas. Prior to the analysis, the compounddependent mass spectrometer parameters of the eight compounds were optimized by infusion. The optimized parameters are listed in 
Table S2. For each compound, two MRM transitions were monitored (Table 2); one transition was used for quantification, whereas the other was used as a qualifier MRM to ensure correct identification. The identity of the compounds was also confirmed by recording full MS/MS spectra in selected bee extracts and comparing these with spectra recorded of authentic analytical standards.

Atropine, gelsemine, senkirkine, senecionine, and methyllycaconitine were analyzed collectively in positive mode. The eluents were A: $7 \%$ acetonitrile in Milli-Q water with $0.5 \%$ formic acid and B: $95 \%$ acetonitrile and 5\% Milli-Q water with $0.5 \%$ formic acid. The compounds were separated on a Synergy Fusion column $(150 \mathrm{~mm} \times$ $2 \mathrm{~mm}, 4 \mu \mathrm{m}$; Phenomenex, Værløse, Denmark) with a flow rate of 0.4 $\mathrm{mL} / \mathrm{min}$, and the gradient was as follows: $0-2 \mathrm{~min}: 100 \% \mathrm{~A} ; 2-18$ min: $100-30 \%$ A; $18-19$ min: $30-0 \%$ A; $19-22$ min: $0 \%$ A; $22-23$ min: $0-100 \%$ A and $23-30$ min: $100 \%$ A. Source parameters were as follows: curtain gas (CUR), 45 psi; collision gas (CAD), medium; temperature (TEM), $400{ }^{\circ} \mathrm{C}$; ion source gas 1 (GS 1), $90 \mathrm{psi}$; ion source gas 2 (GS 2), $30 \mathrm{psi}$; and ionspray voltage (IS), $4200 \mathrm{~V}$. Amygdalin was analyzed using the same source parameters and chromatographic method as described above, but in negative mode. Aucubin was also analyzed in negative mode using the same solvent system and column as described above, but the gradient was as follows: $0-3 \mathrm{~min}: 100 \% \mathrm{~A} ; 3-13 \mathrm{~min}: 100-75 \% \mathrm{~A} ; 13-14 \mathrm{~min}$ : $75-$ 0\% A; $14-17$ min: $0 \%$ A; $17-18$ min: $0-100 \%$ A; and $18-28 \mathrm{~min}$ : $100 \%$ A. From 1 to $14 \mathrm{~min}$, the flow rate was $0.2 \mathrm{~mL} / \mathrm{min}$, whereas from 14 to $28 \mathrm{~min}$, it was increased to $0.3 \mathrm{~mL} / \mathrm{min}$. Source parameters were as follows: CUR, 50 psi; CAD, medium; TEM, 100 ${ }^{\circ} \mathrm{C}$, GS 1, $50 \mathrm{psi}$; GS 2, $50 \mathrm{psi}$; and IS, $-4500 \mathrm{~V}$. Triptolide was analyzed in positive mode as its ammonium adduct, as previously reported by Zhuang et al. ${ }^{32}$ The column was a Hypersil BDS C18 ( $250 \mathrm{~mm} \times 2.1 \mathrm{~mm}, 5 \mu \mathrm{m}$; Thermo Fisher, Hvidovre, Denmark), and the solvent system consisted of $5 \mathrm{mM}$ ammonium formate (A) and methanol (B). The following gradient was applied with a flow rate of $0.3 \mathrm{~mL} / \mathrm{min}: 0-8 \mathrm{~min}: 55 \% \mathrm{~A} ; 8-9 \mathrm{~min}: 55-0 \% \mathrm{~A} ; 9-13 \mathrm{~min}: 0 \% \mathrm{~A}$; 13-14 $\mathrm{min}: 0-55 \% \mathrm{~A}$; and $14-21 \mathrm{~min}: 55 \% \mathrm{~A}$. The source parameters were as follows: CUR, 40 psi; CAD, medium; TEM, 200 ${ }^{\circ} \mathrm{C}$; GS 1, 50 psi; GS 2, 80 psi; and IS, 5000 V. For all analyses, the column temperatures were $30^{\circ} \mathrm{C}$, and the auto sampler was set to 10 ${ }^{\circ} \mathrm{C}$. An injection volume of $30 \mu \mathrm{L}$ was used for the triptolide analyses, whereas 20 and $30 \mu \mathrm{L}$ were injected for the remaining compounds for whole and dissected bees, respectively.

Quantification was performed in SCIEX Analyst version 1.6.2, and the concentration intervals of the recorded standard curves were as follows: $0.00075-25 \mathrm{ppb}$ for methyllycaconitine, $0.003-25 \mathrm{ppb}$ for senkirkine and senecionine, $0.006-100 \mathrm{ppb}$ for amygdalin, atropine, and gelsemine, $6.25-200 \mathrm{ppb}$ for aucubin, and $0.048-12.5 \mathrm{ppb}$ for triptolide.

Calculations and Statistics. Calculations converting the sample extract concentrations exported from the SCIEX Analyst software were performed in Microsoft Excel 2016, which was also used to calculate mean values, SDs, and RSD \%. The pairwise $T$-tests with Bonferroni corrections were performed in $\mathrm{R}$ version 3.2.1.

\section{RESULTS}

Performance of the Analytical Method. EURACHEM guidelines ${ }^{29}$ state that acceptable recovery percentages should be in the range of $80-120 \%$. The recovery percentages from the initial test of the methods $(N=3)$ were within the recommended interval, except for amygdalin that displayed recovery percentages of $78 \%$ (data not shown). Method precision was very high with RSD below 6\%, except for aucubin, which had an RSD of $10 \%$ (data not shown). Upon full method validation with eight replicates, the recovery percentages decreased to $67-108 \%$ (Table 2), which was expected because of the lower concentrations in the extracts for most of the compounds compared with the initial tests. The original data were not corrected for the recovery percentages established above. Precision of the methods was high with RSD \% of $3-13 \%$ (Table 2).

Most bee samples displayed concentrations above the LOD, except for one bee fed on amygdalin and three bees fed on methyllycaconitine, which had concentrations below the LOD. More bee samples displayed concentrations below the LOQ and the numbers of bees fed on each of the eight phytochemicals displaying concentrations below LOQ were as follows: senkirkine, 3; amygdalin, 3; atropine, 1; methyllycaconitine, 6; senecionine, 3 ; aucubin, 7; and triptolide, 4. These samples were nonetheless included in the data set. $^{33}$

Food Intake. The bees' intake of the spiked 50\% sucrose solutions was measured by weighing every $24 \mathrm{~h}$, and the mean consumption of the individual compounds per bee per $24 \mathrm{~h}$ was calculated (Figure 1). No significant differences were

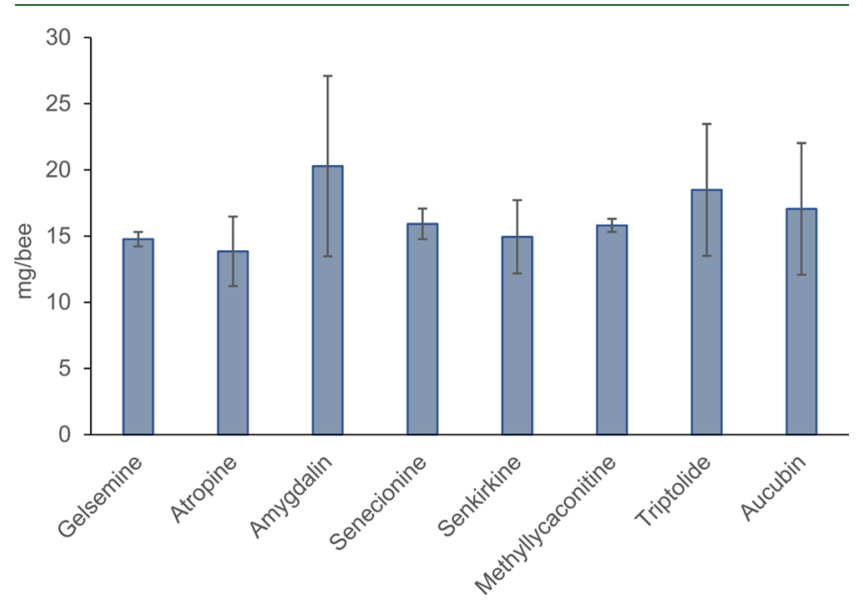

Figure 1. Mean consumption per bee per $24 \mathrm{~h}$ of the $50 \%$ sucrose solutions spiked with the eight phytochemicals during the 5-day feeding period. Error bars represent SDs. There were no significant differences in the food consumption between the groups of bees fed on the individual phytochemicals $(P \gg 0.05$, Pairwise $T$-test with Bonferroni corrected $P$-values).

observed between the groups $(P \gg 0.05$, pairwise $T$-test with Bonferroni corrected $P$-values), suggesting no differences in the bees' preferences for the tested compounds. The mean and total consumption of the individual compounds are listed in Table S3. None of the bees displayed any signs of feeding deterrence and/or intoxication, and there were only few dead bees (1-2 per group).

Concentrations of Phytochemicals in Whole and Dissected Honey Bees. The mean concentrations in the whole bees (Figure 2 and Table S3) corresponded to 6-30\% of the individual bees' mean total consumption of the individual compounds during the whole five-day period or $29-149 \%$ of the individual bees' mean daily consumption rates. The compounds supplied in higher concentrations in the diet generally displayed higher levels in the bees, but this trend was not linear. There were, for instance, differences between the quantities measured in the bees of senkirkine and senecionine, which were supplied in equal concentrations in the sucrose solutions. The concentrations of atropine and amygdalin measured in the bees were almost identical, although the concentrations in the sucrose solutions were 18.6 and $50 \mathrm{ppm}$, respectively. None of the eight compounds were found in the control bees sampled at beginning of the experiment. 
Aucubin

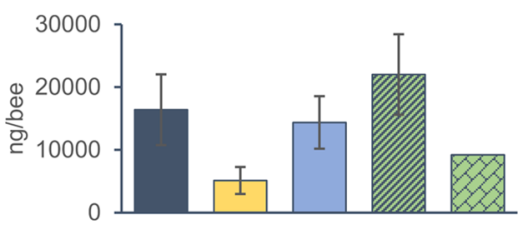

Amygdalin

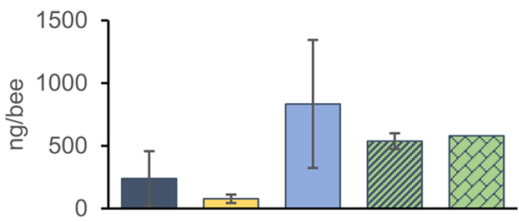

Senkirkine

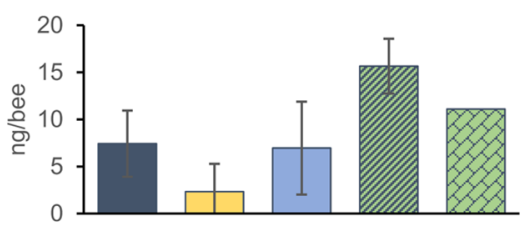

Triptolide

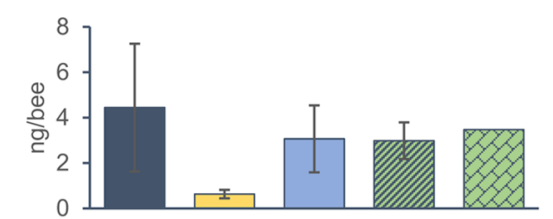

Gelsemine

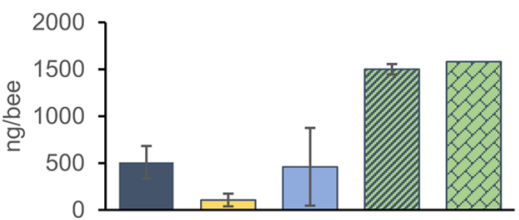

Atropine

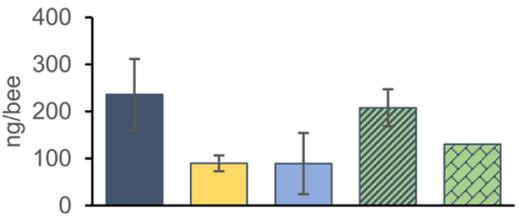

Senecionine

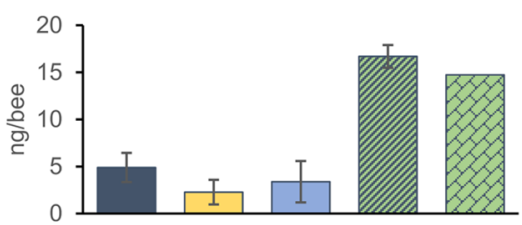

Methyllycaconitine

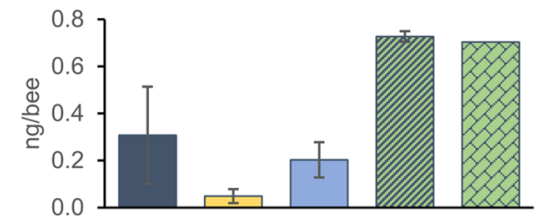

Whole bees $\square$ Guts $\square$ Bees without guts

Mean daily intake Intake day 4-5

Figure 2. Mean concentrations (ng/bee) of the eight phytochemicals quantified in individual whole $(N=12)$ and dissected bees $(N=6)$ sampled on day $5,2 \mathrm{~h}$ after the feeding was terminated. For the dissected bees, the guts were removed and analyzed separately from the rest of the bee (bees without guts) to assess compound distribution within the bees. For each individual dissected bee, the sum of the quantities recovered in the gut and the rest of the bee from which the gut was removed (bees without guts) designates the total quantity of compound recovered in that individual. The concentrations are displayed together with the bees' mean daily food intake and the consumption during the last 24 h of feeding (day 4-5). Error bars represent SDs. The total compound intakes over the whole feeding period (day 1-5) are not shown in the figure; they were as follows (ng/bee): aucubin, 110,020; gelsemine, 7502; amygdalin, 2687; atropine, 1038; senkirkine, 78; senecionine, 84; triptolid, 15; and methyllycaconitine, 4 .

When honey bees are kept in small feeding boxes in darkness and fed on sucrose solutions, they rarely defecate, ${ }^{34}$ and no fecal depositions were observed in the feeding boxes. Thus, compound concentrations in the bees can only diminish via chemical transformations inside the bees. Metabolization percentages can therefore be calculated by comparing the compound quantities recovered in the individual whole bees with the total compound intake per bee during the 5-day experiment (Figure 3A) and the intake per bee during the last $24 \mathrm{~h}$ of feeding (Figure $3 \mathrm{~B}$ ). Of the total compound quantities consumed by the bees during the whole 5-day feeding period, $70-94 \%$ had been metabolized on day 5, $2 \mathrm{~h}$ after the feeding was terminated (Figure 3A). The metabolization percentages of atropine and triptolide were significantly lower $(P<0.05$; pairwise $T$-test with Bonferroni-corrected $P$-values) compared to the other compounds. Comparisons of the quantities recovered in the bees with the quantities consumed during the last $24 \mathrm{~h}$ of feeding revealed metabolization percentages between -81 and $68 \%$. The negative metabolization percentages observed for some compounds originate from the bees' compound consumption being higher than their $24 \mathrm{~h}$ metabolization. The metabolization percentages of atropine, triptolide, and aucubin during the last $24 \mathrm{~h}$ of feeding were significantly lower compared with the other compounds $(P<$ 0.05 ; pairwise $T$-test with Bonferroni-corrected $P$-values) (Figure 3B).

In the individual dissected bees $(N=6)$, the sum of the quantities recovered in the guts and the bees without guts designates the total quantity of compound recovered in that individual. The compound distribution between the gut and the bee without gut was calculated as the percentage of compound recovered in the guts and bees without guts, respectively (Figure 4). Concentrations measured in the dissected bees are displayed in Figure 2 and Table S4. Significantly higher percentages $(P<0.05$; pairwise $T$-test with Bonferroni-corrected $P$-values) of senkirkine, amygdalin, methyllycaconitine, and triptolide were present in the bees without gut compared with the guts. The mean percentages of senecionine and gelsemine were also higher in the bees without guts compared with the guts, but these differences were nonsignificant $(P>0.05)$. Aucubin and atropine were present in higher percentages in the guts, although the distribution was 
A: Metabolization day 1-5

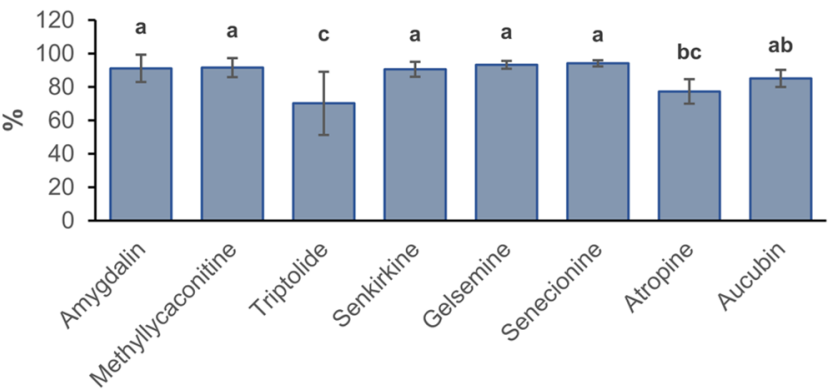

B: Metabolization day 4-5

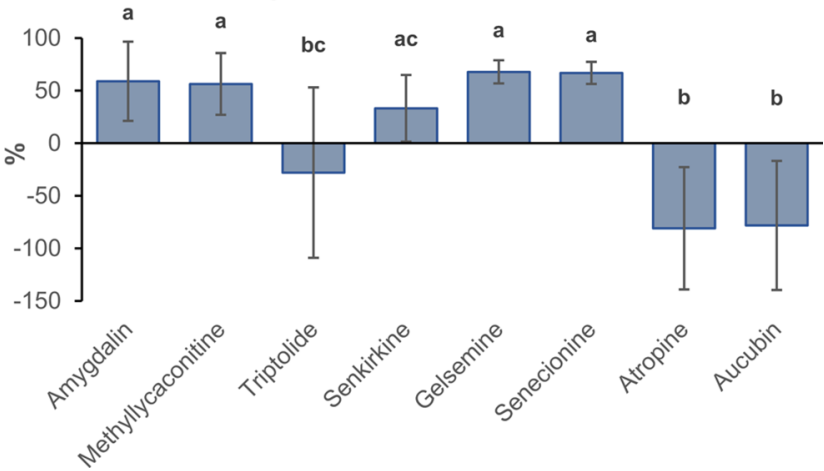

Figure 3. (A) Metabolization percentages of the individual phytochemicals from day 1 to 5 . The percentages were calculated by comparing the mean consumption rates per bee with the mean concentrations measured in the individual bees $(N=12)$ sampled on day $5,2 \mathrm{~h}$ after the feeding was terminated. (B) Metabolization percentages of the individual phytochemicals from day 4 to 5 . The percentages were calculated by comparing the mean consumption rates per bee during the last $24 \mathrm{~h}$ of feeding with the mean concentrations measured in the individual bees $(N=12)$ sampled on day $5,2 \mathrm{~h}$ after the feeding was terminated. Error bars represent SD. Bars that do not share similar letters denote statistically significant differences $(P<0.05)$ determined by a pairwise $T$-test with Bonferroni-adjusted $P$-values.

significant only for aucubin $(P<0.05$; pairwise $T$-test with Bonferroni-corrected $P$-values) (Figure 4$)$.

Oral bioavailability is calculated as the percentage of the ingested compound quantities during the 5-day feeding period recovered in the bees without guts. The bioavailability ranged from $4 \%$ for senecionine to $31 \%$ for amygdalin (Table 3 ).

Table 3. Oral Bioavailability of the Eight Phytochemicals Calculated as the Percentage of the Total Ingested Quantity Over the 5-Day Feeding Period Recovered in the Bees Without Guts ${ }^{a}$

\begin{tabular}{lc}
\hline \multicolumn{1}{c}{ compound } & oral bioavailability (\%) \\
amygdalin & $31.0 \pm 19.0$ \\
triptolide & $20.6 \pm 9.9$ \\
aucubin & $13.1 \pm 3.8$ \\
senkirkine & $8.9 \pm 6.3$ \\
atropine & $8.6 \pm 6.3$ \\
gelsemine & $6.1 \pm 5.5$ \\
methyllycaconitine & $5.6 \pm 2.1$ \\
senecionine & $4.0 \pm 2.6$
\end{tabular}

${ }^{a}$ The percentages are listed \pm the SD.

\section{DISCUSSION}

Studies of Phytochemicals and Bees. The eight phytochemicals included in this feeding experiment were singled out for their phytochemical properties and have been included in other experiments with honey and/or bumble bees. Gelsemine, aucubin, and amygdalin were tested in experiments focusing on health-promoting effects. Aucubin significantly stimulated honey bee immune functions, ${ }^{18}$ whereas both gelsemine and aucubin significantly decreased the levels of the bumble bee gut protozoan Crithidia bombi. ${ }^{22,35}$ Gelsemine in higher concentrations can, however, negatively affect bumble bee fecundity, ${ }^{36}$ yet similar effects have not been shown in the solitary bee Osmia lignaria, ${ }^{37}$ and its effect on honey bee fecundity has not been established. Toxic and/or deterrent effects have been reported for amygdalin $\left(\mathrm{LD}_{50}=\right.$ $0.003 \%),{ }^{20,38,39}$ atropine $\left(\mathrm{LD}_{50}=0.1 \%\right),{ }^{20}$ and triptolide, ${ }^{27,40}$ as well as pyrrolizidine alkaloids ${ }^{34}$ such as senecionine and senkirkine. The concentrations fed to the bees in this experiment were below toxicity thresholds. Atropine consumed in concentrations above $10^{-2} \mathrm{M}$ can even impair honey bee memory retrieval. ${ }^{41}$ For the pyrrolizidine alkaloids, certain structural features have been linked to their toxicity, especially the 1,2-double bond, which is present in both of the pyrrolizidine alkaloids, senecionine and senkirkine. ${ }^{34}$ Neither

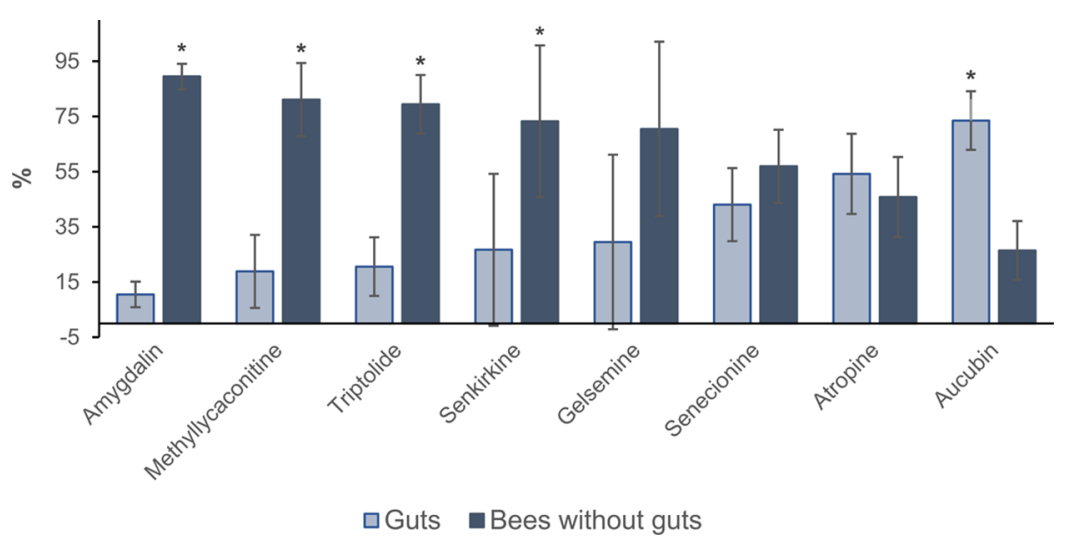

Figure 4. Mean distribution of the eight phytochemicals between the gut and the rest of the bee from which the gut was removed (bees without guts) measured in the dissected bees $(N=6)$. Error bars represent $S D$. Asterisks indicate significant differences $(P \leq 0.05$; pairwise $T$-test with Bonferoni-corrected $P$-values) between the percentages recovered in the guts and bees without guts for each of the individual phytochemicals. 
toxicity nor beneficial effects have been reported for methyllycaconitine, but this phytochemical is a known nicotine acetylcholine receptor antagonist used in experiments. ${ }^{42}$ The nicotine acetylcholine receptor is also the target site for neonicotinoids, which, in contrast, function as receptor agonists. ${ }^{43}$ The combined effects of honey bee exposure to both neonicotinoids and methyllycaconitine have not been investigated.

Concentrations of Phytochemicals in Honey Bees. The eight selected phytochemicals were recovered in both whole and dissected bees, but large variations in the compound concentrations were observed between the individual bees for all phytochemicals. The RSDs in percent (RSD \%) were 3292 and $19-128 \%$ for whole and dissected bees, respectively (Tables S3 and S4). The bees continuously had access to the fortified sucrose solutions, and it was not possible to monitor the feeding patterns of each individual bee. If one bee feeds on the sucrose solution immediately after it is provided, it results in a longer time period, during which metabolic breakdown of the compound can occur compared to another bee that did not feed until hours later. When bees are kept in groups, trophallaxis (exchange of liquids between colony members) results in food being distributed between individuals. ${ }^{44}$ This behavior further complicates accurate estimation of the food intake and specific time of feeding for each individual within the group. Complex hierarchies also develop in small groups of bees, affecting exchange of food. ${ }^{45}$ Thus, the feeding patterns are the most plausible explanation for the concentration differences measured in the individual bees for each of the phytochemicals. The experimental conditions nonetheless mimic the conditions in the hive, and similar variations between individuals would be expected in field-collected honey bees.

Phytochemical Metabolization. Only a fraction of the consumed quantities of all eight phytochemicals were recovered in the bees on day 5 (Figure 2). Plant secondary metabolites are xenobiotics to bees, and upon ingestion, a series of enzymatic detoxification mechanisms will commence. ${ }^{46,47}$ Because the bees did not defecate in the small feeding boxes, the only possible way the compound concentrations can diminish is via biotransformations within the bees. Therefore, ample metabolic breakdown of all eight compounds must have occurred. Our data also show significantly different metabolization rates of the individual compounds. Comparisons of the quantities ingested during the whole 5-day feeding period with the quantities recovered in the bees reveals significantly lower metabolization percentages of atropine and triptolide (Figure 3A). Comparisons with the amounts ingested during the last $24 \mathrm{~h}$ of feeding augmented these effects. For this comparison, atropine, triptolide, and aucubin displayed significantly lower metabolization percentages (Figure 3B). Higher quantities of atropine, aucubin, and triptolide than the bees had consumed during the last $24 \mathrm{~h}$ of feeding were measured in the bees, resulting in negative metabolization percentages. This demonstrates that the bees are not capable of metabolizing the quantities they have consumed within $24 \mathrm{~h}$, resulting in temporary compound accumulation (Figures 2 and 3B). The tested compounds can therefore be divided into two groups, with senkirkine, senecionine, gelsemine, methyllycaconitine, and amygdalin being metabolized significantly faster relative to atropine, aucubin, and triptolide. The faster metabolism demonstrated for senkirkine and senecionine in conjunction with their lower oral bioavailability $(<10 \%$, Table 3$)$ may be part of the reason why honey bees seemingly thrive on plants producing pyrrolizidine alkaloids despite the known toxicity of several of these compounds. Honey bees are attracted to many plants producing pyrrolizidine alkaloids such as Borago officinalis, Symphytum spp., and Echium spp., of which some are even important plants for bees. ${ }^{48-50}$ However, the faster metabolism could also have a negative impact because pyrrolizidine alkaloids having the 1,2-double bond, such as senecionine and senkirkine, are considered pro-toxic because the toxic intermediate is formed through bioactivations by cytochrome P450 enzymes. ${ }^{34}$ In contrast to many specialist insects ${ }^{34}$ and a solitary bee, ${ }^{51}$ the generalist honey bee does not seem to have developed any specific strategies to cope with pyrrolizidine alkaloids. To fully understand this conundrum, detailed studies of pyrrolizidine alkaloid fate and metabolism in bees, which have not yet been conducted, are required. The faster metabolism established for amygdalin is supported by the previously demonstrated enzyme activity directed toward the degradation of cyanogenic glycosides, such as amygdalin, in honey bees. ${ }^{38}$ The slower metabolization rates of atropine, aucubin, and triptolide are unlikely to result from a delayed feeding response caused by a deterrent effect of these compounds because of the 5 days of feeding and the bees' need to take up sugar daily. ${ }^{52}$ Additionally, no signs of deterrent effects were observed during our regular monitoring of the bees throughout the experiment. Neither were any significant differences in the consumption of sucrose solutions fortified with these compounds observed (Figure 1). Hence, we conclude that differences in the chemical properties and structure of the individual phytochemicals are the underlying cause of the observed differences in metabolization. Senkirkine and senecionine are structurally and chemically similar compounds (Table 1), both belonging to the group of phytochemicals displaying faster metabolism, and this result supports our conclusion. Amygdalin and aucubin are both glycosylated compounds which could readily be cleaved into their respective aglycons by omnipresent glycosidase enzymes, yet only amygdalin belongs to the faster metabolizing group of compounds. This may at least in part be explained by the significantly higher concentration in which aucubin was provided compared to amygdalin (Table 1).

Oral Bioavailability and Phytochemical Uptake from the Gut. Many insects utilize dietary phytochemicals as defense compounds and sequester these from their diets. ${ }^{53}$ Thus, transport of phytochemicals from insect guts into their haemolymph, individual tissues, and organs is known. However, there is a paucity of information regarding gut uptake and oral bioavailability of dietary plant secondary metabolites in honey bees. For dietary bioactive phytochemicals to have an impact on honey bee health, such as anti-viral and/or immunostimulatory activity, uptake from the gut seems a prerequisite. Our results clearly demonstrate gut uptake of all eight phytochemicals because the compounds were recovered in the dissected bees after the guts had been removed (bees without guts, Figure 4). Amygdalin has previously been found in the haemolymph of honey bees after ingestion of dietary amygdalin, ${ }^{54}$ and that result supports our findings. Besides gut uptake, our results also prove clear differences in the uptake of the individual compounds (Figure 4). Aucubin was the only compound with a significantly higher percentage remaining in the gut (Figure 4), but the oral bioavailability is nonetheless the third highest among the eight compounds (13\%, Table 3 ). 
Aucubin was supplied in the highest concentrations in the food (1600 ppm), and the ingested quantities may exceed the bees' uptake capabilities. In another experiment, aucubin feeding significantly increased the expression of the antimicrobial peptide, hymenoptaecin, in honey bees. ${ }^{18}$ Together with other antimicrobial peptides, hymenoptaecin is an intrinsic part of the honey bee immune system. ${ }^{55}$ Our demonstration of gut uptake of aucubin supports that the intact form of this molecule is indeed available in the bees' body and thus can impact honey bee immune functions.

The different rates of uptake can be ascribed to the chemical properties and the structure of the individual compounds or a combination of these factors. Detailed investigations of the uptake kinetics of the individual compounds should be performed in future experiments to clarify this. Senkirkine, amygdalin, methyllycaconitine, and triptolide were present in significantly higher percentages in the bees without guts, as compared to the guts (Figure 4). Amygdalin and triptolide displayed the highest oral bioavailability (percentage of ingested phytochemical taken up from the gut; 31 and $21 \%$, respectively, Table 3 ), whereas senkirkine and methyllycaconitine were below $10 \%$. These compounds were supplied in very different concentrations ranging from 0.057 to $126 \mathrm{ppm}$. Thus, no clear trend for oral bioavailability could be related to compound concentrations in the food. Amygdalin was, for instance, present in the diet at $50 \mathrm{ppm}$ but displayed the highest oral bioavailability and the lowest percentage in the guts $(11 \%)$. Gelsemine and senkirkine were supplied in the sucrose solutions at 126 and $1.3 \mathrm{ppm}$, respectively, but the percentages remaining in the guts were almost identical (gelsemine: $30 \%$; senkirkine: $27 \%$ ), and the oral bioavailability was comparable (gelsemine: 6\%; senkirkine: 9\%), again suggesting that compound concentrations in the diet have limited influence on the gut uptake. The two highly similar pyrrolizidine alkaloids, senkirkine and senecionine, were supplied in the same concentrations (1.3 ppm). Despite this, differences in distribution percentages and oral bioavailability were evident. Senkirkine was present in significantly higher concentrations in the bees without guts, whereas senecionine was distributed almost equally between the guts and bees without guts (guts: 43\%; bees without guts: 57\%, Figure 4). This is also reflected in the oral bioavailability of the two compounds (senkirkine 9\%; senecionine 4\%, Table 3). These results underline that the bees' compound uptake from the gut is associated more with the chemical properties and structure of the individual compounds and less with the concentrations supplied in the food. It also shows that even small differences in the structure can influence uptake and oral bioavailability. In mammals, it is well established that compound uptake from the intestine is influenced by the compound structure and properties. $^{56-58}$ For instance, methylated flavones are more effectively absorbed than demethylated flavones. ${ }^{56}$ Similarly, the results of our experiment suggest the hypothesis that analogous mechanisms influence gut uptake in honey bees. In mammals, some compounds cannot be taken up from the intestine in their original form but require chemical transformation in the gut before uptake can occur. ${ }^{59}$ In our experiment, no metabolites were targeted. Although the results clearly demonstrate that all compounds were taken up by the bees in their original form, it cannot be excluded that chemical transformations occurs in the gut and that metabolites are also taken up. This should be evaluated in future studies.
Factors Influencing the Fate of Dietary Phytochemicals. Honey bees harbor a range of microorganisms in their guts, ${ }^{60}$ which may be involved in the breakdown of dietary xenobiotics. ${ }^{61}$ It has also been established that honey bees can regulate the rate of passage of food from the crop to the midgut, ${ }^{62}$ and insects are known to possess chemosensory mechanisms for the detection of dietary toxins in their crops and midguts. ${ }^{63}$ In insects, including bees, the midgut is the site for digestion of food and nutrient absorption, ${ }^{64}$ but it has not yet been clarified if dietary phytochemicals are also taken up in this part of the bees' digestive system. Both gut microbiota and regulatory mechanisms may impact the quantities of dietary phytochemicals amenable for uptake and thereby influence the oral bioavailability of bioactives. Such mechanisms may be responsible for the oral bioavailability of the eight compounds not exceeding $31 \%$. The gut microorganisms may also function in conjunction with the honey bees' enzymatic detoxification system and be partly responsible for the observed phytochemical breakdown.

In comparison with many other insects, honey bees have a deficit in all gene families controlling their enzymatic detoxification of xenobiotics. ${ }^{46}$ Thus, it has been proposed that honey bees may exploit gut microbes and behavioral mechanisms complementing their enzymatic detoxification such as selective foraging, dilution of xenobiotics by mixing different types of pollen/nectar, as well as the cultivation of hive microorganisms involved in the conversion of pollen into bee bread. ${ }^{46}$ In this experiment, behavioral factors can be excluded because the bees were offered a no-choice diet under controlled conditions. Nonetheless, ample degradation of the dietary phytochemicals was observed. Thus, under simple experimental conditions, we can conclude that honey bees are highly capable of metabolizing the phytochemicals offered individually. However, the involvement of gut microbiota should be clarified in future experiments, as should potential synergistic effects between individual compounds. More complex compound mixtures should gradually be introduced in future studies to unravel the metabolic apparatus of the honey bees. Quercetin and p-coumaric acid are almost omnipresent phytochemical constituents of pollen/nectar and capable of upregulating the expression of xenobiotic-metabolizing cytochrome P450 genes in honey bees. ${ }^{14,15,65}$ Thus far, the effects of $p$-coumaric acid and quercetin have only been studied on the detoxification of pesticide xenobiotics, and changes in pesticide toxicity ${ }^{14,65}$ as well as in residual concentrations have been reported. ${ }^{66}$ This underlines the need to complement uptake/metabolization studies of phytochemicals in honey bees with a natural pollen and nectar diet. Certain compounds may also inhibit the enzymatic degradation capabilities of honey bees. This has been reported for triazole fungicides that inhibit cytochrome P450 monooxygenases, which are also involved in metabolizing dietary phytochemicals. ${ }^{67}$ Phytochemicals with similar modes of action may well exist.

In summary, the honey bees' pollen/nectar diet is a complex mixture of xenobiotics, and studies of especially natural xenobiotics are in the early stages and many aspects still need to be clarified. With this experiment, we demonstrate effective metabolization of a variety of phytochemicals in honey bees. The metabolization rate differed among the phytochemicals, with atropine, aucubine, and triptolide being metabolized significantly slower than senecionine, senkirkine, amygdalin, methyllycaconitine, and gelsemine. Gut uptake of 
dietary phytochemicals is also clearly demonstrated, and this underlines the potential for phytochemicals to impact honey bee physiology and health. The highest oral bioavailability of $31 \%$ was observed for amygdalin, whereas the lowest of $4 \%$ was for senecionine (amygdalin $>$ triptolide $>$ aucubin $>$ senkirkine $>$ atropine $>$ gelsemine $>$ methyllycaconitine $>$ senecionine). Variations in the concentrations of the compounds supplied in the food did not seem to significantly affect the results. Instead, we conclude that the observed differences in metabolization, gut uptake, and oral bioavailability are due to differences in the structure and properties of the selected phytochemicals. In future studies, particularly the involvement of gut microorganisms in phytochemical breakdown as well as synergistic effects between phytochemicals should be targeted, together with detailed kinetics studies to fully understand the honey bees' metabolic apparatus.

\section{ASSOCIATED CONTENT}

\section{s) Supporting Information}

The Supporting Information is available free of charge at https://pubs.acs.org/doi/10.1021/acs.jafc.0c03584.

Botanical sources of the eight phytochemicals fed to honey bees, compound-dependent mass spectrometer parameters, phytochemical intake and mean phytochemical concentrations measured in the whole bees, and phytochemical concentrations measured in the dissected bees (PDF)

\section{AUTHOR INFORMATION}

\section{Corresponding Author}

Nanna Hjort Vidkjær - Department of Biology and Biological Engineering, Chalmers University of Technology, SE-412 96 Göteborg, Sweden; Department of Agroecology, Aarhus University, DK-4200 Slagelse, Denmark; 이이.org/00000001-6383-7674; Email: nanna.vidkjaer@chalmers.se, nanna.vidkjaer@gmail.com

\section{Authors}

Inge S. Fomsgaard - Department of Agroecology, Aarhus University, DK-4200 Slagelse, Denmark; 이잉.org/00000003-4987-8511

Per Kryger - Department of Agroecology, Aarhus University, DK-4200 Slagelse, Denmark

Complete contact information is available at:

https://pubs.acs.org/10.1021/acs.jafc.0c03584

\section{Author Contributions}

Conceptualization, N.H.V.; methodology, N.H.V., I.S.F., and P.K.; feeding experiment, N.H.V. and P.K.; chemical analyses, N.H.V.; data analyses, N.H.V.; manuscript writing and preparation, N.H.V. (P.K. and I.S.F. provided feedback); project administration, N.H.V.; funding acquisition, N.H.V.

\section{Funding}

This work was funded by an independent postdoc grant for N.H.V. from the Danish Counsel for Independent Research for the project: "Arming honey bees with nature's pharmacy" (DFF-6111-00441).

\section{Notes}

The authors declare no competing financial interest.

\section{ACKNOWLEDGMENTS}

The authors would like to thank Roy Mathew Francis for providing the honey bee photographs used in the table of content graphics and Bente B. Laursen for valuable and skilled assistance during the experiments.

\section{ABBREVIATIONS}

MRM, multiple reaction monitoring mode; LOD, limit of detection; LOQ limit of quantitation; RSD, relative standard deviation; SD, standard deviation

\section{REFERENCES}

(1) Gallai, N.; Salles, J.-M.; Settele, J.; Vaissière, B. E. Economic valuation of the vulnerability of world agriculture confronted with pollinator decline. Ecol. Entomol. 2009, 68, 810-821.

(2) Genersch, E. Honey bee pathology: Current threats to honey bees and beekeeping. Appl. Microbiol. Biotechnol. 2010, 87, 87-97.

(3) Goulson, D.; Nicholls, E.; Botias, C.; Rotheray, E. L. Bee declines driven by combined stress from parasites, pesticides, and lack of flowers. Science 2015, 347, 1255957.

(4) Nicholls, C. I.; Altieri, M. A. Plant biodiversity enhances bees and other insect pollinators in agroecosystems. A review. Agron. Sustainable Dev. 2013, 33, 257-274.

(5) Vanbergen, A. J.; Initiative, t. I. P.; Biesmeijer, J. C.; Britton, N. F.; Brown, M. J. F.; Brown, M.; Bryden, J.; Budge, G. E.; Bull, J. C.; Carvell, C.; et al. Threats to an ecosystem service: Pressures on pollinators. Front. Ecol. Environ. 2013, 11, 251-259.

(6) Berenbaum, M. R. Does the honey bee "risk cup" runneth over? Estimating aggregate exposures for assessing pesticide risks to honey bees in agroecosystems. J. Agric. Food Chem. 2016, 64, 13-20.

(7) Chen, Y. P.; Siede, R. Honey bee viruses. In Advances in Virus Research, 1st ed.; Maramorosch, K., Shatkin, A. J., Murphy, F. A., Eds.; Elsevier Academic Press: USA, 2007; Vol. 70, pp 33-80.

(8) McMenamin, A. J.; Brutscher, L. M.; Glenny, W.; Flenniken, M. L. Abiotic and biotic factors affecting the replication and pathogenicity of bee viruses. Curr. Opin. Insect. Sci. 2016, 16, 14-21.

(9) Sánchez-Bayo, F.; Goulson, D.; Pennacchio, F.; Nazzi, F.; Goka, K.; Desneux, N. Are bee diseases linked to pesticides? - a brief review. Environ. Int. 2016, 89-90, 7-11.

(10) Di Pasquale, G.; Salignon, M.; Le Conte, Y.; Belzunces, L. P.; Decourtye, A.; Kretzschmar, A.; Suchail, S.; Brunet, J.-L.; Alaux, C. Influence of pollen nutrition on honey bee health: Do pollen quality and diversity matter? PLoS One 2013, 8, No. e72016.

(11) Dolezal, A. G.; Toth, A. L. Feedbacks between nutrition and disease in honey bee health. Curr. Opin. Insect. Sci. 2018, 26, 114119.

(12) Frias, B. E. D.; Barbosa, C. D.; Lourenço, A. P. Pollen nutrition in honey bees (Apis mellifera): Impact on adult health. Apidologie 2016, 47, 15-25.

(13) Maistrello, L.; Lodesani, M.; Costa, C.; Leonardi, F.; Marani, G.; Caldon, M.; Mutinelli, F.; Granato, A. Screening of natural compounds for the control of Nosema disease in honeybees (Apis mellifera). Apidologie 2008, 39, 436-445.

(14) Johnson, R. M.; Mao, W.; Pollock, H. S.; Niu, G.; Schuler, M. A.; Berenbaum, M. R. Ecologically appropriate xenobiotics induce cytochrome p450s in Apis mellifera. PLoS One 2012, 7, No. e31051.

(15) Mao, W.; Schuler, M. A.; Berenbaum, M. R. Honey constituents up-regulate detoxification and immunity genes in the western honey bee Apis mellifera. Proc. Natl. Acad. Sci. U.S.A. 2013, $110,8842-8846$.

(16) Negri, P.; Maggi, M. D.; Ramirez, L.; De Feudis, L.; Szwarski, N.; Quintana, S.; Eguaras, M. J.; Lamattina, L. Abscisic acid enhances the immune response in Apis mellifera and contributes to the colony fitness. Apidologie 2015, 46, 542-557.

(17) Aurori, A. C.; Bobiș, O.; Dezmirean, D. S.; Mărghitaș, L. A.; Erler, S. Bay laurel (Laurus nobilis) as potential antiviral treatment in naturally BQCV infected honeybees. Virus Res. 2016, 222, 29-33. 
(18) Palmer-Young, E. C.; Tozkar, C. Ö.; Schwarz, R. S.; Chen, Y.; Irwin, R. E.; Adler, L. S.; Evans, J. D. Nectar and pollen phytochemicals stimulate honey bee (Hymenoptera: Apidae) immunity to viral infection. J. Econ. Entomol. 2017, 110, 1959-1972.

(19) Bernklau, E.; Bjostad, L.; Hogeboom, A.; Carlisle, A.; Arathi, H. S. Dietary phytochemicals, honey bee longevity and pathogen tolerance. Insects 2019, 10, 14.

(20) Detzel, A.; Wink, M. Attraction, deterrence or intoxication of bees (Apis mellifera) by plant allelochemicals. Chemoecology 1993, 4, $8-18$.

(21) Irwin, R. E.; Cook, D.; Richardson, L. L.; Manson, J. S.; Gardner, D. R. Secondary compounds in floral rewards of toxic rangeland plants: Impacts on pollinators. J. Agric. Food Chem. 2014, 62, 7335-7344.

(22) Manson, J. S.; Otterstatter, M. C.; Thomson, J. D. Consumption of a nectar alkaloid reduces pathogen load in bumble bees. Oecologia 2010, 162, 81-89.

(23) Martinello, M.; Cristofoli, C.; Gallina, A.; Mutinelli, F. Easy and rapid method for the quantitative determination of pyrrolizidine alkaloids in honey by ultra performance liquid chromatography-mass spectrometry: An evaluation in commercial honey. Food Control 2014, 37, 146-152.

(24) Boros, B.; Farkas, Á.; Jakabová, S.; Bacskay, I.; Kilar, F.; Felinger, A. LC-MS quantitative determination of atropine and scopolamine in the floral nectar of Datura species. Chromatographia 2010, 71, 43-49.

(25) Richardson, L. L.; Bowers, M. D.; Irwin, R. E. Nectar chemistry mediates the behavior of parasitized bees: Consequences for plant fitness. Ecology 2016, 97, 325-337.

(26) London-Shafir, I.; Shafir, S.; Eisikowitch, D. Amygdalin in almond nectar and pollen - facts and possible roles. Plant Syst. Evol. 2003, 238, 87-95.

(27) Tan, K.; Guo, Y. H.; Nicolson, S. W.; Radloff, S. E.; Song, Q. S.; Hepburn, H. R. Honeybee (Apis cerana) foraging responses to the toxic honey of Tripterygium hypoglaucum (Celastraceae): Changing threshold of nectar acceptability. J. Chem. Ecol. 2007, 33, 2209-2217.

(28) Cook, D.; Manson, J. S.; Gardner, D. R.; Welch, K. D.; Irwin, R. E. Norditerpene alkaloid concentrations in tissues and floral rewards of larkspurs and impacts on pollinators. Biochem. Syst. Ecol. 2013, 48, $123-131$.

(29) Eurachem Guide: The Fitness for Purpose of Analytical MethodsA Laboratory Guide to Method Validation and Related Topics, 2nd ed.; Magnusson, B., Örnemark, U., Eds.; Eurachem, 2014.

(30) Wang, Z.; Wen, J.; Xing, J.; Guo, D. Determination of triptolide and triptonide in human plasma by high-performance liquid chromatography. Biomed. Chromatogr. 2005, 19, 60-62.

(31) Carreck, N. L.; Andree, M.; Brent, C. S.; Cox-Foster, D.; Dade, H. A.; Ellis, J. D.; Hatjina, F.; van Englesdorp, D. Standard methods for Apis mellifera anatomy and dissection. J. Apic. Res. 2013, 52, 140.

(32) Zhuang, X.-M.; Liu, P.-X.; Zhang, Y.-J.; Li, C.-K.; Li, Y.; Wang, J.; Zhou, L.; Zhang, Z.-Q. Simultaneous determination of triptolide and its prodrug $\mathrm{mc002}$ in dog blood by LC-MS/MS and its application in pharmacokinetic studies. J. Ethnopharmacol. 2013, 150, $131-137$.

(33) Analytical Method Committee. Recommendations for the definition, estimation and use of the detection limit. Analyst 1987, 112, 199-204.

(34) Reinhard, A.; Janke, M.; von der Ohe, W.; Kempf, M.; Theuring, C.; Hartmann, T.; Schreier, P.; Beuerle, T. Feeding deterrence and detrimental effects of pyrrolizidine alkaloids fed to honey bees (Apis mellifera). J. Chem. Ecol. 2009, 35, 1086-1095.

(35) Richardson, L. L.; Adler, L. S.; Leonard, A. S.; Andicoechea, J.; Regan, K. H.; Anthony, W. E.; Manson, J. S.; Irwin, R. E. Secondary metabolites in floral nectar reduce parasite infections in bumblebees. Proc. R. Soc. B 2015, 282, 20142471.

(36) Manson, J. S.; Thomson, J. D. Post-ingestive effects of nectar alkaloids depend on dominance status of bumblebees. Ecol. Entomol. 2009, 34, 421-426.
(37) Elliott, S. E.; Irwin, R. E.; Adler, L. S.; Williams, N. M. The nectar alkaloid, gelsemine, does not affect offspring performance of a native solitary bee, Osmia lignaria (Megachilidae). Ecol. Entomol. 2008, 33, 298-304.

(38) Lecocq, A.; Green, A.; Pinheiro De Castro, É.; Olsen, C.; Jensen, A.; Zagrobelny, M. Honeybees tolerate cyanogenic glucosides from clover nectar and flowers. Insects 2018, 9, 31.

(39) Singaravelan, N.; Nee'man, G.; Inbar, M.; Izhaki, I. Feeding responses of free-flying honeybees to secondary compounds mimicking floral nectars. J. Chem. Ecol. 2005, 31, 2791-2804.

(40) Liu, X.; Zhou, Y.; Li, Y.; Tang, L.; Luo, P.; Wen, S. Determination of triptolide in honey by ultra performance liquid chromatography-tandem mass spectrometry. J. Food Saf. Food Qual. 2016, 7, 4960-4965.

(41) Lozano, V. C.; Gauthier, M. Effects of the muscarinic antagonists atropine and pirenzepine on olfactory conditioning in the honeybee. Pharmacol., Biochem. Behav. 1998, 59, 903-907.

(42) Dacher, M.; Gauthier, M. Involvement of no-synthase and nicotinic receptors in learning in the honey bee. Physiol. Behav. 2008, 95, 200-207.

(43) Casida, J. E. Neonicotinoids and other insect nicotinic receptor competitive modulators: Progress and prospects. Annu. Rev. Entomol. 2018, 63, 125-144.

(44) Brodschneider, R.; Libor, A.; Kupelwieser, V.; Crailsheim, K. Food consumption and food exchange of caged honey bees using a radioactive labelled sugar solution. PLoS One 2017, 12, No. e0174684.

(45) Biganski, S.; Kurze, C.; Müller, M. Y.; Moritz, R. F. A. Social response of healthy honeybees towards Nosema ceranae-infected workers: Care or kill? Apidologie 2018, 49, 325-334.

(46) Berenbaum, M. R.; Johnson, R. M. Xenobiotic detoxification pathways in honey bees. Curr. Opin. Insect. Sci. 2015, 10, 51-58.

(47) Gong, Y.; Diao, Q. Current knowledge of detoxification mechanisms of xenobiotic in honey bees. Ecotoxicology 2017, 26, 112.

(48) Denisow, B. Flowers ecology and pollen output of Symphytum officinale L. J. Apic. Sci. 2008, 52, 81-89.

(49) Thom, M. D.; Eberle, C. A.; Forcella, F.; Gesch, R.; Weyers, S.; Lundgren, J. G. Nectar production in oilseeds: Food for pollinators in an agricultural landscape. Crop Sci. 2016, 56, 727-739.

(50) Martín Arroyo, T.; González-Porto, A. V.; Bartolomé Esteban, C. Viper's bugloss (Echium spp.) honey typing and establishing the pollen threshold for monofloral honey. PLoS One 2017, 12, No. e0185405.

(51) Praz, C. J.; Müller, A.; Dorn, S. Specialized bees fail to develop on non-host pollen: Do plants chemically protect their pollen? Ecology 2008, 89, 795-804.

(52) Mayack, C.; Naug, D. Starving honeybees lose self-control. Biol. Lett. 2015, 11, 20140820.

(53) Opitz, S. E. W.; Müller, C. Plant chemistry and insect sequestration. Chemoecology 2009, 19, 117-154.

(54) Hurst, V.; Stevenson, P. C.; Wright, G. A. Toxins induce "malaise" behaviour in the honeybee (Apis mellifera). J. Comp. Physiol., A 2014, 200, 881-890.

(55) Danihlík, J.; Aronstein, K.; Petřivalský, M. Antimicrobial peptides: A key component of honey bee innate immunity physiology, biochemistry, and chemical ecology. J. Apic. Res. 2015, 54, 123-136.

(56) Wen, X.; Walle, T. Methylated flavonoids have greatly improved intestinal absorption and metabolic stability. Drug Metab. Dispos. 2006, 34, 1786-1792.

(57) Walle, T. Absorption and metabolism of flavonoids. Free Radical Biol. Med. 2004, 36, 829-837.

(58) Laerke, H. N.; Mortensen, M. A.; Hedemann, M. S.; Bach Knudsen, K. E.; Penalvo, J. L.; Adlercreutz, H. Quantitative aspects of the metabolism of lignans in pigs fed fibre-enriched rye and wheat bread. Br. J. Nutr. 2009, 102, 985-994.

(59) Graefe, E. U.; Wittig, J.; Mueller, S.; Riethling, A.-K.; Uehleke, B.; Drewelow, B.; Pforte, H.; Jacobasch, G.; Derendorf, H.; Veit, M. Pharmacokinetics and bioavailability of quercetin glycosides in humans. J. Clin. Pharmacol. 2001, 41, 492-499. 
(60) Kwong, W. K.; Moran, N. A. Gut microbial communities of social bees. Nat. Rev. Microbiol. 2016, 14, 374-384.

(61) Kešnerová, L.; Mars, R. A. T.; Ellegaard, K. M.; Troilo, M.; Sauer, U.; Engel, P. Disentangling metabolic functions of bacteria in the honey bee gut. PLoS Biol. 2017, 15, No. e2003467.

(62) Blatt, J.; Roces, F. Haemolymph sugar levels in foraging honeybees (Apis mellifera carnica): Dependence on metabolic rate and in vivo measurement of maximal rates of trehalose synthesis. $J$. Exp. Biol. 2001, 204, 2709-2716.

(63) Park, J.-H.; Kwon, J. Y. Heterogeneous expression of Drosophila gustatory receptors in enteroendocrine cells. PLoS One 2011, 6, No. e29022.

(64) Terra, W. R.; Ferreira, C. Biochemistry and molecular biology of digestion. In Insect Molecular Biology and Biochemistry, 1st ed.; Gilbert, L. I., Ed.; Academic Press: London, 2012; pp 365-418.

(65) Wong, M. J.; Liao, L.-H.; Berenbaum, M. R. Biphasic concentration-dependent interaction between imidacloprid and dietary phytochemicals in honey bees (Apis mellifera). PLoS One 2018, 13, No. e0206625.

(66) Ardalani, H.; Vidkjær, N. H.; Laursen, B. B.; Kryger, P.; Fomsgaard, I. S. Dietary quercetin impacts the concentration of pesticides in honey bees. Chemosphere 2021, 262, 127848.

(67) Mao, W.; Schuler, M. A.; Berenbaum, M. R. Disruption of quercetin metabolism by fungicide affects energy production in honey bees (Apis mellifera). Proc. Natl. Acad. Sci. U.S.A. 2017, 114, 25382543.

(68) Kempf, M.; Heil, S.; Haßlauer, I.; Schmidt, L.; von der Ohe, K.; Theuring, C.; Reinhard, A.; Schreier, P.; Beuerle, T. Pyrrolizidine alkaloids in pollen and pollen products. Mol. Nutr. Food Res. 2010, 54, 292-300. 